\title{
Aplicación de la psicología al estudio de los procedimientos policiales de recuerdo de personas
}

\author{
José Joaquín Mira* \\ Margarita Diges
}

Universidad Autónoma Madrid

Este trabajo se sitúa dentro del tema más general de «Memoria de Personas», un área de la Psicología de reciente denominación (Hastie y cols., 1980), aunque sus orígenes pueden remontarse a fines del siglo XIX con la aparición de las primeras investigaciones sobre la memoria de testigos de sucesos (Diges y Mira, 1983).

Por «Memoria de Personas» se entiende un cuerpo de investigaciones y modelos teóricos que pretenden explicar cómo se adquiere, organiza, guarda y recupera la información sobre las personas que conocemos o encontramos en la vida diaria, por lo que en su mismo objeto de estudio se determina ya una confluencia e interacción de distintos enfoques psicológicos: desde la perspectiva cognitiva - con que se abordan en la actualidad los procesos de memoria y pensamiento-, al enfoque social -característico de la interacción personal y la moderna cognición social-, pasando por los modelos de personalidad y del self - propios de una Psicología preocupada por las diferencias individuales-, y por el enfoque más aplicado de la Psicología del Testimonio.

Todo esto hace que la «Memoria de Personas» sea un campo en el que tanto las teorías como las técnicas de investigación se diversifican de tal modo que resulta necesario sistematizar y delimitar los distintos campos de explicación y acción. Este esquema clarificador del área no se ha llevado a cabo aún, pero aquí vamos a utilizar uno bastante elemental para centrar este trabajo.

Así, desde un punto de vista teórico, los temas que se pueden poner bajo el rótulo de «Memoria de Personas» se sitúan en tres grandes apartados:

1. La adquisición de información sobre personas y eventos sociales con ellas relacionados.

2. La retención de información sobre personas -que implica su organización y la definición de reglas de relación e inferencia para tratarla- y sobre los sucesos que les ocurren (conductas o acciones).

3. La utilización o recuperación de la información anterior, que puede ser implícita o explícita. 
Dentro de este último apartado y específicamente en el caso de la recuperación explícita, es donde cabe hablar de procedimientos policiales de identificación de personas. Por recuperación explícita se entiende la utilización de información de memoria acerca de personas y sucesos relacionados, siempre y cuando la utilización sirva a un fin expreso, deliberado o consciente. Implica tanto el reconocimiento como el recuerdo de la apariencia física, la identidad, características personales y/o las acciones de las personas; pueden incluirse aquí, también, los juicios sobre las personas y acciones que realizan cuando deliberadamente se basan en, o requieren, un acto de recuperación a partir de la memoria.

Dado que la tarea de un testigo interrogado por la policía para identificar a un sospechoso consiste, habitualmente, en recordar y/o reconocer a las personas implicadas en un suceso, por lo general delictivo, y en recordar el propio suceso, queda claro que el testigo debe hacer un esfuerzo de recuperación consciente y deliberado de cuanto ha visto.

Por otro lado, desde un punto de vista metodológico conviene hacer una distinción entre los trabajos experimentales y los trabajos que se basan en la actuación real de los testigos en la vida cotidiana. En los primeros se ha recurrido, fundamentalmente, a diseños que minimicen la influencia de variables extrañas a la hora de examinar los factores que influyen en el mejor o peor recuerdo y/o reconocimiento, pero los resultados contradictorios, así obtenidos, han llevado a pensar que esta misma metodología está afectando e invalidando las conclusiones de muchos trabajos, hasta el punto de que ha llegado a considerarse que el problema más importante con que se enfrenta la Psicología del Testimonio es, precisamente, la rigidez de la metodología empleada (Mira y Diges, 1984). En cuanto a los trabajos basados en casos reales, es obvio que su principal desventaja provieñe de la falta de control experimental. No obstante, sus resultados sí que se corresponden con el del funcionamiento real de las personas en una situación social en la que existen ciertas demandas de memoria. Por último, hay que resaltar que estas investigaciones tienen un interés social intrínseco, puesto que la actuación de una persona como testigo de un hecho delictivo puede tener consecuencias importantes para la persona o personas implicadas en el hecho y, también, para el propio testigo.

Desde esta perspectiva, en este artículo vamos a considerar principalmente aquellas investigaciones de "corte real», obviando aquellas otras de «corte experimentalista».

\section{IDENTIFICACION DE PERSONAS}

Es clásica dentro del área de la Psicología del Testimonio la distinción de los procedimientos policiales de identificación de personas -realizada por Clifford y Bull en su libro The Psychology of Person Identification (1978) - en función de la tarea que implican:

1. Sistemas de reconocimiento, en los que un testigo procede a la identificación de una persona que ha visto antes entre un grupo de sujetos que ahora está viendo, como ocurre con la práctica en comisarías de lo que se denomina «rueda de presos», en donde un testigo intenta identificar a un sospechoso entre diversos individuos. 
2. Sistemas de recuerdo, en los que al testigo se le pide la recomposición o descripción de una persona vista con anterioridad; ejemplos muy conocidos son el "retrato-robot», o el menos sofisticado «dibujo a carboncillo».

En un trabajo anterior (Mira y Diges, 1983) hemos realizado una evaluación psicológica de los procedimientos de reconocimiento de personas que se utilizan en diversos paises por los servicios policiales correspondientes, así como de las variables que influyen en mayor o menor grado en la fiabilidad de ese reconocimiento; por lo que el tema central de este artículo serán los procedimientos que la policía utiliza (o dispone) para identificar a un sospechoso basándose en el recuerdo que de él tienen los testigos.

Los estudios de Psicología sobre este tema abarcan:

1. La descripción de los sistemas que la policía utiliza para interrogar a los testigos y que se basen en la descripción del sospechoso según el recuerdo que de él tenga el testigo.

2. Qué estudios hay sobre la eficacia y utilidad de estos sistemas.

3. Sistemas más avanzados, desarrollados a partir de trabajos experimentales paralelos al trabajo policial.

4. Qué recomendaciones, desde un punto de vita psicológico, pueden darse a la policía sobre el uso de estos procedimientos.

\section{SISTEMAS DE RECUERDO}

El primer sistema de recuerdo de caras data de 1880 y no fue, precisamente, ideado para ese fin, sino para que los policías pudieran recordar mejor la cara de los delincuentes que debían buscar y así encontrarlos con más facilidad por las calles. Su uso, sin embargo, fue muy diferente al comprobarse su utilidad a la hora de interrogar a testigos sobre cómo era el sospechoso.

En la actualidad, los sistemas de recuerdo de caras tienen en común: a) que en todos la cara está dividida en sus distintas partes (ojos; pelo, barbilla, cejas, boca, labios, nariz, oreja...); b) que el operador-policía es un experto que ha recibido entrenamiento especializado para utilizar los sistemas de recuerdo y puede, o no, ser la persona que interroga a los testigos; c) que el procedimiento habitual consiste en combinar, por ensayo y error, las características faciales que el testigo cree que son semejantes a las de la persona buscada, comenzando primero por el tipo de peinado y la forma de los ojos y terminando al retocar la imagen final para obtener un parecido más exacto.

En cambio, los sistemas de recuerdo se diferencian entre sí en cuanto a: a) el número de características faciales disponibles en cada sistema; b) el número de accesorios disponibles (barbas, gafas, sombreros, cicatrices...); c) los diferentes soportes o modos de presentación (papel, cartón, televisión, fotografías, etc...); d) el tiempo necesario para especializarse en su uso; e) el tiempo que se tarda en componer la imagen de un sospechoso; f) las facilidades dadas a los testigos para que seleccionen los rasgos más semejantes al original; g) la calidad de la imagen final de la persona. 
Los sistemas de recuerdo de caras más utilizados por la policía son los siguientes:

A) Dibujos a carboncillo. Es el instrumento más socorrido y de menor costo si exceptuamos al profesional que lo realiza, siendo precisamente esto su mayor inconveniente debido a la amplia experiencia necesaria para llevarlo a cabo. Al desarrollarse otros sistemas más sofisticados se pensó, en un primer momento, que estos dibujos perderían utilidad. Sin embargo, la tendencia actual es verlos como un complemento útil de otros sistemas de recuerdo (Davies, 1981, 1982).

B) Identi-Kit. Este sistema data de 1950 y pertenece a la familia de los «retratos-robot» diseñados originalmente en Francia. Consiste en una-serie de láminas transparentes en las que aparecen impresas diferentes características faciales (130 tipos de peinados diferentes, 102 ojos, 37 narices, 40 labios y 52 barbillas distintas); cuenta, además, con un surtido de otras características y accesorios como cejas, cicatrices, gafas, arrugas para la piel, barbas, sombreros, etc., también variados, que permiten completar la imagen de la persona buscada. Este sistema tiene, sin embargo, el inconveniente de no estar preparado para reproducir caras de mujeres o de varones que no sean de raza blanca, lo que limita su empleo.

La composición de una cara se logra superponiendo las transparencias elegidas por el testigo, por ensayo y error, acercándose a la imagen que él cree fiel a la realidad y modificando posteriormente tanto la longitud de la cara como otros detalles adicionales con un lápiz de tinta china. Todos los elementos están codificados para facilitar su comunicación, empleo y búsqueda*.

C) Pbotofit. Es el sistema de identificación que ha sustituido al anterior por su mayor calidad. Pertenece también a la categoría de «retratos-robot». Los componentes del Photofit son fotografías de características faciales impresas en cartulinas. Cuenta con 195 tipos de peinados y orejas, 99 ojos y cejas, 89 narices, 105 bocas, 74 barbillas y mejillas distintas y diferentes cartulinas con barbas, gafas, cicatrices, etc. Este sistema está preparado para ser utilizado en el caso de que la persona buscada sea mujer, o de raza asiática o negra. Al igual que en el caso anterior, cualquier detalle de última hora puede ser dibujado por el operador en una transparencia y añadido al original. Existe también una codificación facial para facilitar su uso, ideada por el creador del sistema, Jacques Penry (1971), con la ventaja de que además del código aparece una fotografía a escala reducida de la característica facial de que se trate, en un Indice Visual que facilita enormemente la tarea al testigo.

Su modo de empleo es igual que en el caso anterior*.

\section{Sistemas por ordenador}

Sólo existen a nivel experimental y con carácter de investigación. Los más desarrollados son los siguientes:

* Para mayor información sobre el Identi-Kit, consultar Davies, 1982.

A raiz de la mala comercialización del Identi-Kit ha salido al mercado el Identi-Kit II que lleva impresas fotos en vez de dibujos y que cuenta con un mayor número de transparencias. 
F) Whatsisface System (Gillenson y Chandrasekaran, 1975). Este sistema tiene almacenadas en su memoria 17 características faciales diferentes, siete de las cuales están combinadas con otra distinta, lo que multiplica las posibilidades de cambio. Una persona no entrenada puede utilizar el sistema de recuerdo siguiendo las instrucciones del propio ordenador.

G) Sketch System. Este sistema es un desarrollo del anterior al serle intercalado un display entre el conjunto del Whatsisface System y el usuario para que éste pueda hablar con el sistema, aunque con un lenguaje muy reducido.

H) Wiederbold. Al sistema Minolta Montage Synthetizer (MMS) se le acopló en 1976, con carácter experimental, un ordenador (Wiederhold, 1976; citado en Laughery, Rhodes y Rotten, 1980) que reproduce las fotografías del MMS en una matriz de $128 \times 128$, con diferentes grados de luminosidad, en un monitor y que se denomina igual que su inventor.

\section{FIABILIDAD DE LOS SISTEMAS DE RECUERDO}

Los estudios sobre la eficacia de los sistemas de recuerdo que utiliza la policía para obtener reproducciones de la cara de las personas están, en la mayoría de los casos, alejados de las imprescindibles condiciones que asegurarán la validez ecológica de las investigaciones (Malpass y Devine, 1981). Las condiciones son extremadamente artificiales, en muy pocas ocasiones los investigadores han salido de sus laboratorios, o han empleado caras reales (Patterson, 1978; Mira, 1983). Por ello aquí, nos referiremos únicamente a los estudios de las universidades de Aberdeen y Houston, responsables de la mayoría de los trabajos de esta área, de los cuales puede decirse que existe ligazón con las necesidades reales de la policía.

Los datos de que se dispone sobre los sistemas de recuerdo de la familia de los retratos-robot no son concluyentes, aunque parece que al comparar los tres sistemas entre sí, el uso de dibujos es más recomendable que el empleo del Identi-Kit (Laughery y Fowler, 1980; Laughery y Smith, 1978) - hay que tener presente que no se disponen de datos sobre el Identi-Kit II-; y que al comparar los dibujos a carboncillo con el Photofit, este último parece resultar más eficaz (Ellis, Davies y Shepherd, 1978).

Con respecto a la comparación Identi-Kit vs. Photofit, la práctica diaria habla de un abandono del primero en favor del Photofit, lo que muestra que el segundo, a juicio de las policías de distintos países, opera con mayor calidad.

En una encuesta a policías escoceses para conocer la utilidad y eficacia del sistema Photofit en situaciones reales, las fuerzas de seguridad valoraron este sistema de identificación de personaŝ como sigue:

Photofit enteramente responsable de la resolución del caso ........... 5\% Photofit muy útil en la resolución del caso ........................................17\%

Photofit útil en la resolución del caso................................................33\%

Photofit no demasiado útil en la resolución del caso..........................20\%

Photofit nada útil en la resolución del caso.........................................25\% 
Datos semejantes habían sido publicados cuatro años antes por el mismo grupo de investigadores (Davies, Ellis y Shepherd, 1978) resaltando que si bien en más casos es útil que ineficaz, en bastantes investigaciones policiales el Photofit es completamente inútil, quizá por las condiciones de estrés de los testigos que, al mismo tiempo, son la víctima. El problema está -entonces- en poder especificar qué testigos son los que recuerdan mejor y en qué circunstancias los recuerdos son fidedignos, por un lado; y conocer las limitaciones de los sistemas de identificación de personas, en cuanto a su mismo diseño, por otra.

Centrándonos en el Photofit - que parece ser el sistema de recuerdo más eficaz- se ha podido constatar que los errores que cometen los testigos a la hora de componer el retrato-robot son consistentes más que aleatorios, y que se deben a concepciones estereotipadas que los testigos poseen sobre cómo son los delincuentes. En este sentido, es reveladora la conclusión de Shepherd, Ellis, McMurran y Davies (1978) de que la percepción de los atributos físicos se ve poderosamente afectada por los valores sociales.

Al mismo tiempo, se han estudiado los efectos de otras variables sobre la calidad del retrato-robot final. La principal de estas variables sometidas a estudio ha sido el intervalo de demora entre el suceso y el interrogatorio por parte de la policía. En los estudios realizados, el intervalo de tiempo entre un delito y la composición de un retrato-robot no ha tenido ningún efecto sobre la calidad de la imagen, siempre que se realizara en el plazo máximo de una semana (Davies, Ellis y Shepherd, 1978).

Otras variables estudiadas son: la posible interferencia al ver distintas formas de las mismas características faciales (por ejemplo, 20 tipos de ojos) y tener que escoger sólo una, que parece no afectar significativamente a la imagen final (Davies y Christie, 1982); el modo de presentación, por medio de fotos o de dibujos, donde parece que ciertas rayas de los retratos-robot disminuyen la calidad de la imagen final, y los retoques son imprescindibles (Davies, 1982); la representatividad de las características faciales con que cuenta el sistema, donde el Photofit aventaja a los otros sistemas (Davies, 1982); la importancia de la restauración del contexto en que ocurrió el suceso durante el interrogatorio en la comisaría, para guiar la memoria del testigo y mejorar, así, la calidad de su recuerdo (Davies y Milne, 1985); el tener presente estudios anteriores que llaman la atención sobre la posibilidad de presiones, sobre los testigos, para que se produzca una respuesta, para poder llevar adelante el trabajo policial (Buckhout, 1974); y la posibilidad, intimamente unida a la anterior, de que una vez formada la imagen del sospechoso $-\mathrm{y}$ aunque no sea la correcta - el testigo se autoconvenza de la autenticidad de su recuerdo y malogre el trabajo policial (Loftus, 1976; Mauldin y Laughery, 1981).

Con respécto a la construcción de los sistemas de retrato-robot, una importantísima limitación es, precisamente, que estos sistemas entienden que la cara de una persona debe componerse sumándose las diferentes características faciales aisladas, no interpretándose como un todo (Davies y Christie, 1982). Sin embargo, parece que la percepción y la memoria humanas se adecúan mejor a una interpretación global del todo (Christie y Ellis, 1981; Clifford y Bull, 1978).

En cuanto a los sistemas de rompecabezas y los sintetizadores ópticos $-\mathrm{y}$ aunque se están utilizando por las policías de Alemania Federal, Italia, Francia, Estados Unidos y Japón- los estudios experi- 
mentales sobre su utilidad son escasos, a excepción de los realizados sobre el Field Identification System en la Universidad de Houston. Respecto a este último, los datos son desalentadores debido a que en este sistema las rayas horizontales de los trazos desnaturalizan la cara de la persona buscada (Yount y Laughery, 1982; Laughery, Smith y Yount, 1981).

Por último, sobre la eficacia de los sistemas de identificación por ordenador, sólo podemos decir que no existen aún datos experimentales que avalen su mayor eficacia frente a los sistemas tradicionales, puesto que en su mayor parte se encuentran en fase de desarrollo. Pese a esto, se tienen fundadas esperanzas - puesto que en su diseño superan muchas de las deficiencias de los retratos-robot- de que en el futuro sean los sistemas de mayor uso (Laughery, Rhodes y Batten, 1981).

\section{RESUMEN Y CONCLUSIONES}

Hace más de cien años que la policía se vale de instrumentos construidos con la finalidad de confeccionar un rostro que se parezca al de la persona involucrada en sucesos de su competencia. Los carteles anunciando la recompensa por algún delincuente - popularizados en las películas del oeste americano- son un buen ejemplo y el antecedente más inmediato de los modernos sistemas de identificación de personas basados en el recuerdo de los testigos.

Todos estos instrumentos, conocidos como «retratos-robot», se basan en las capacidades de memoria de los sujetos-testigos pero, sin embargo, se han diseñado a espaldas de los estudios sobre el funcionamiento de la memoria humana que, desde la psicología, se han venido realizando en este último siglo y, en especial, de las investigaciones que bajo el rótulo «Psicología del Testimonio» se han llevado a cabo sobre el comportamiento de los testigos y la eficacia de los sistemas de recuerdo de caras que la policía utiliza.

En este artículo hemos intentado condensar los estudios realizados sobre los procedimientos policiales de recuerdo de personas a nuestro juicio más relevantes, puesto que se desarrollaron simulando las condiciones reales lo más exactamente posible, resaltando la capacidad de la ciencia psicológica en un campo «relativamente» nuevo y, a todas luces, aplicado. Al hacerlo así, nos hemos visto obligados a obviar gran número de experimentos sobre memoria de caras o material con significado - muchos de los cuales conocerá el lector- debido a que no cumplían estos requisitos, pero aportando - creemos- una visión más acertada, al hacerlo así, de lo que se entiende por Psicología del Testimonio.

Delimitemos, primero, el área; segundo, describimos los procedimientos policiales más difundidos en los diferentes países; $y$, en tercer lugar, comentamos los resultados de las investigaciones psicológicas sobre los instrumentos genéricamente llamados «retratos-robot» y que, principalmente, han sido realizados en las universidades de Aberdeen (Escocia) y Houston (USA). Los datos más relevantes son:

- que existen una caras que son más fáciles de recordar que otras (Laughery y Fowler, 1980; Shepherd y Ellis, 1973);

- que hay sistemas que están mejor preparados para un tipo de caras que para otro y que, cuando coinciden, el resultado es siem- 
pre el «mejor posible artificialmente» (Laughery y Fowler, 1980);

- que existe el peligro real de lo que se denomina transferencia inconsciente (Loftus, 1976) y de que estos procedimientos de recuerdo, previos a ruedas de presos, distorsionen la información almacenada en la memoria del testigo y lleven a falsos positivos (Mauldin y Laughery, 1981);

- que la estrategia de selección característica a característica no es útil y, por tanto, los sistemas de ordenador con monitor de televisión que ofrecen una visión de la cara creada, en todo momento, almacenando los cambios realizados hasta entonces, parecen más idóneos (Laughery, Rhodes y Batten, 1981). En el mismo sentido, algunos autores han sugerido como alternativa que la policía trabaje con un álbum de fotos de caras sobre las cuales el testigo elige una que se parezca algo a la de la persona buscada $y$, posteriormente, sobre esa foto, se hacen variaciones que siempre permitirán una aproximación más holista (Yount y Laughery, 1982), igual que se sugería antes con el empleo de ordenadores;

- que los estudios sobre recuerdo se han realizado sobre todo entre las universidades de Aberdeen y Houston y esto, precisamente, por la conexión de estos investigadores con la policía local, colaboración que no está presente en otros muchos casos y que, obviamente resulta fundamental dado el carácter aplicado de esta rama de la Psicología;

- que, unido al punto anterior, se observa una desconfianza por parte de las Instituciones responsables de la policía en la credibilidad del trabajo del psicólogo en muchos países (Devlin, 1976);

- que los sistemas de retrato-robot parecen ser útiles, tan sólo, en un 50 por 100 de las investigaciones policiales (Davies, 1982);

- que de entre todos los sistemas - si no se tienen en cuenta los computerizados por encontrarse en fase de desarrollo- parece que el Photofit es el que ofrece mejores posibilidades, seguido de los sistemas de rompecabezas, muy utilizados por las policías europeas.

Tras esta exposición, creemos que - al menos - dos cosas deben haber quedado aclaradas:

1. La utilidad de la Psicología en aquellas tareas que de algún modo son de su competencia; como ocurre en este caso con el comportamiento de testigos y policías y con la medida de las capacidades cognitivas de los individuos, en este caso testigos.

2. La importancia de diseños experimentales cercanos a la realidad para fundamentar el desarrollo de áreas de la Psicología, como ésta del Testimonio, de una evidente urgencia y presión social de resultados. 


\section{Referencias}

BRown, J., y MONK, D. «Individual Differences in the Relation of Recognition to Recall», en Gruneberg, Morris y Sykes (Eds.): Practical Aspects of Memory. Academic Press, Londres, 1978.

BucKHOUT, R. «Eyewitness Testimony». Scientific American, 1974, 231, 23-31.

Clifford, B. y BULL, R. The Psychology of Person Identification. Londres. Routledge and Kegan Paul, Ltd., 1978.

Christie, D., y EluIs, H. «Photofit construction versus verbal descriptions of faces». Jo. of Applied Psycho$\log y, 1981$, vol. 66 (3), 358-363.

DavIES, G. «Face Recall Systems», en Davies, Ellis \& Shepherd (Eds.): Perceiving and Remembering Faces. Academic Press, 1981.

DAvIES, G. «Composite Systems for Recalling Faces-Helping the Police With Their Enquiries?», en Trankell (Ed.): Reconstructing the Past: The Role of Psychologists in Criminal Trials. Norstedts, Estocolmo, 1982.

Davies, G., y Christie, D. «Face Recall: An Examination of Some Factors Limiting Composite Production». Jo. Applied Psychology, 1982, vol. 67, n. ${ }^{\circ}$ 1, 103-109.

Davies, G.; Ellis, H., y SHEPHERD, «Face Identification: The Influence of Delay Upon Accuracy of Photofit Construction». Jo. Police Science and Administration, 1978, vol. 6, n. ${ }^{9} 1$.

Davies, G., y Mil.ne, A. «Eyewitness Composite Production. A function of mental or physical reinstatement of context». Criminal Justice and Behavior, 1985, 12, 2, 209-220

DEVIIN REPORT. «Report to the Secretary of State for the Home Department of the Departmental Committee on Evidence of Identification in Criminal Cases». HMSO, Londres, 1976.

Diges, M., y MiRA, J. J. «Psicólogos, Psicología y Testimonio». Análisis y Modificación de conducta, 1983, 9, 21, 243-270.

Ellis, H.; Davies, G., y SHEPherd, J. «A Critical Examination of the Photofit System for Recalling Faces». Ergonomics, 1978, 21, 297-307.

Gillenson, M., y Chandrasekaran, B. «A Heuristic Strategy for Developing Human Facial Images on a CRT». Pattern Recognition, 1975, 7, 187-196.

Hastie, R.; Ostrom, T. M.; Ebbesen, E. B.; Wyer, R. S.; Hamilton, D., y Carlston, D. L. Person Memory: The Cognitive Basis of Social Perception, Hillsdale, N. J. LEA, 1980.

Laughery, K., y Fowler, R. «Sketch Artist and Identi-kit Procedures for Recalling Faces». Jo. Applied Psychology, 1980, 65, 307-316.

LAUGHERY, K.; RHODES, B., y BATTEN, G. «Computer-Guided Recognition and Retrieval of Facial Images». Perceiving and Remembering Faces, 1981.

LAUGHery, K.; Smith, V., y Yount, M. «Visual Suport Devices: Evaluation of a new Technique for Constructing Facial Images». Manuscrito no publicado. Universidad de Houston, 1980.

LaUGHerY, K., y SMITH, V. «Suspect Identification Following Exposure to Sketch and Identi-kit Composites». Proceedings of the Human Factors Society 22nd Annual Meeting, Detroit, octubre de 1978.

LoFTUS, E. «Unconscious transference in eyewitness identification». Law \& Psychology Review, 1976, vol. 2, 93-98.

MalPaSS, R., y Devine, P. «Realism and Eyewitness Identification Research». Law \& Human Behavior, 1981, vol. 4, 347-358.

Mauldin, M., y Laughery, K. «Composite Production Effects on Subsequent Facial Recognition». Jo. Applied Psychology, 1981, vol. 66, n.9 3, 351-357.

Mira, J. Memoria en la vida diaria: Un estudio experimental sobre testimonio. Fondo de Publicaciones de la Obra Social de la Caja de Ahorros de Alicante y Murcia, 1985. Microficha. (Tesis de Licenciatura leída el 2 de mayo de 1983.)

MirA, J., y Diges, M. «Tamaño, Sesgos e Imparcialidad de las ruedas de presos». I Congreso de Psicología de la Excepcionalidad, Barcelona, 1983 (en prensa).

Mira, J., y Diges, M. «Psicología del Testimonio: Un problema metodológico». Rev. Psicología General y Aplicada, 1984, 39, 1059-1074.

PATTERSON, K. «Person Recognition: more than a pretty face». Practical Aspects of Memory, 1978.

PENRY, J. Looking at faces and remembering them: A Guide to Facial Identification. Londres: Elek Books, 1971.

SHEPHERD, J., y Ellis, H. «The Effects of Atractiveness on Recognition Memory for Faces. American Jo. Psychology", 1973, vol. 86, n.9 3, 627-633.

ShePherd, J.; Ellis, H.; McMurran, M., y Davies, G. «Effects of Character Attribution on Photofit Construction of a Face». European Jo. Social Psychology, 1978, vol. 8, 263-268.

Yount, M., y Laughery, K. «Facial Memory: Constructing Familiar and Unfamiliar Faces». Bulletin of the Psychonomics Society, 1982, vol. 19 (2), 80-82. 